\title{
Ponto de amostragem ao longo do fuste para estimativa do poder calorífico da madeira
}

\author{
Point of sampling along the shaft for estimate of the calorific power of the wood
}

\author{
Dimas Agostinho da Silva ${ }^{\mathrm{I}}$ Braulio Otomar Caron ${ }^{\mathrm{I}}{ }^{*}$ Alexandre BehlingIII \\ Velci Queiróz de Souza ${ }^{\mathrm{IV}}$ Elder EloyI
}

\section{RESUMO}

Este trabalho teve como objetivo verificar a variação do poder calorífico superior (PCS) ao longo do fuste das espécies florestais Acacia mearnsii De Wild, Eucalyptus grandis W. Hill, Mimosa scabrella Benth. e Ateleia glazioveana Baill e também avaliar o número e as posições das amostras ao longo do fuste para a estimativa do PCS dessas espécies. Para tanto, foram retirados discos de aproximadamente dois centímetros de espessura, ao longo de $0 \%, 25 \%$, $50 \%$ e $75 \%$ da altura total de árvores com um ano de idade para determinação do PCS em bomba calorimétrica (C5000, IKA WORKS). Para Eucalyptus grandis e Ateleia glazioveana, é possível estimar o PCS a partir de um disco, respectivamente, nas posições 50\% e 0\% da altura total, para Mimosa scabrella em dois discos, nas posições $0+75 \%$ e para Acacia mearnsii em três discos, nas posições $0+25+75 \%$ da altura total.

Palavras-chave: bionergia, espécies florestais, floresta energética.

\section{ABSTRACT}

This research had the objective of studing the variation of the calorific power along the shaft of the species forest Acacia mearnsii de Wild, Eucalyptus grandis W. Hill, Mimosa scabrella Benth. and Ateleia glazioveana Baill and also to evaluate the number and the positions of the samples along the shaft for the estimate of calorific power of these species. For so much, they were solitary disks of approximately two centimeters of thickness, along 0\%, 25\%, 50\% and 75\% of the total height of trees, with a year of age, for determination of the power calorific superior in calorimeter (C5000, IKA WORKS). For the Eucalyptus grandis and the Ateleia glazioveana it is possible to esteem the power calorific superior starting from a disk, respectively in the positions $50 \%$ and $0 \%$, and for the Mimosa scabrella with two disks, in the positions $0+75 \%$ and for the Acacia mearnsii, three disks, in the positions $0+25+75 \%$.

Key words: bionergy, forest species, energy forest.

\section{INTRODUÇÃO}

Entre as variáveis que são utilizadas para as estimativas dos parâmetros para avaliar a qualidade da madeira para fins energéticos, além do seu potencial de produção de biomassa, o poder calorífico superior (PCS) tem sido umas das mais estudadas. Para tanto, existe a norma NBR 8633 daABNT que define a maneira de como realizar tal análise, porém, não menciona como proceder com a amostragem da madeira.

Os métodos de amostragem utilizados por diversos pesquisadores têm sido diferentes, o que pode ser constatado em diversos trabalhos. Por exemplo, VALE et al. (2000), comparando a produção de energia da madeira de Eucalyptus grandis W. Hill e a de Acacia mangium Willd, utilizaram amostra composta de cinco posições ao longo do fuste, a $0 \%$, $25 \%, 50 \%, 75 \%$ e $100 \%$ da altura comercial. Já, VALE et al. (2002), avaliando a energia de espécies do cerrado, utilizaram amostras compostas de cinco posições, a

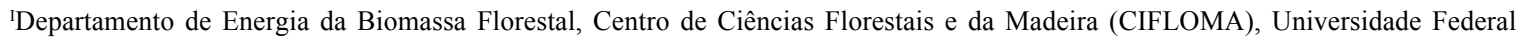
do Paraná (UFPR), Curitiba, PR, Brasil.

"Departamento de Agroclimatologia, Centro de Educação Superior Norte do Rio Grande do Sul (CESNORS), Universidade Federal de Santa Maria (UFSM), 98400-000, Frederico Westphalen, RS, Brasil. E-mail: braulio.caron@pq.cnpq.br.*Autor para correspondência.

IIIDepartamento de Inventário Florestal, Biomassa e Carbono, CIFLOMA, UFPR, Curitiba, PR, Brasil.

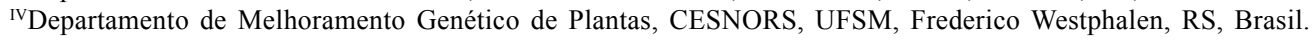


$0 \%, 25 \%, 50 \%, 75 \%$ e $100 \%$ da altura do tronco. PEREIRA \& HIGA (2003), estudando as propriedades da madeira de Taxodium distichum (L.) Rich., entre elas o PCS, utilizaram amostras compostas de discos a cada 2,80m, desde a base até o topo da árvore. CINTRA (2009), avaliando diversas espécies florestais, utilizou amostra composta de discos oriundos de três posições, a $0 \%, 50 \%$ e $100 \%$ da altura do tronco.

Tais divergências encontradas na literatura, muitas vezes, confundem uma proposta para adoção de um ou outro método de amostragem, pois, para cada caso antes apresentado, são os pesquisadores que julgam ser a melhor condição para o seu propósito de estudo. A determinação da maneira de como realizar a amostragem é uma questão de grande importância, pois, ao utilizar amostras de grande tamanho, aumentam-se os custos e o tempo envolvido, ao passo, que muito pequenas, podem levar à grande erro na estimativa da verdadeira média da população.

Nesse sentido, este trabalho vem colaborar para minimizar os tantos métodos de amostragem descritos na literatura. Diante disso, este trabalho teve dois objetivos: o primeiro foi verificar a variação do poder calorífico superior ao longo do fuste das espécies florestais Acacia mearnsii De Wild, Eucalyptus grandis W. Hill, Mimosa scabrella Benth. e Ateleia glazioveana Baill e o segundo foi avaliar o número e a posição das amostras para a estimativa do PCS dessas espécies.

\section{MATERIAL E MÉTODOS}

As amostras utilizadas para a determinação do PCS foram oriundas do experimento que está sendo conduzido com o objetivo de avaliar a produção de madeira para a obtenção de recursos dendroenergéticos, o qual é constituído das espécies Acacia mearnsii, Eucalyptus grandis, Mimosa scabrella e Ateleia glazioveana, submetidas a diferentes condições de espaçamento, conforme relatado em CARON et al. (2011). O experimento está localizado em área pertencente ao Laboratório de Agroclimatologia, vinculado à Universidade Federal de Santa Maria, Campi CESNORS (Centro de Educação Superior Norte do Rio Grande do Sul), sob coordenadas geográficas de $27^{\circ} 22^{\prime \prime} \mathrm{S} ; 53^{\circ} 25^{\prime}$ ' W, a $480 \mathrm{~m}$ de altitude, no município de Frederico Westphalen - RS.

Segundo a classificação climática de Köppen, o clima da região é Cfa. Frederico Westphalen está distante de Iraí à aproximadamente $30 \mathrm{~km}$, sendo o município tomado como referência para os dados de classificação climática. Conforme proposta de MALUF (2000), Iraí apresenta clima de tipo subtemperado subúmido, sendo a temperatura média anual de $18,8^{\circ} \mathrm{C}$ e temperatura média do mês mais frio de $13,3^{\circ} \mathrm{C}$.
A coleta das amostras foi realizada em 2009, um ano após o plantio, conforme cronograma estabelecido pelo projeto. De 12 árvores submetidas ao espaçamento $3,0 \times 1,5 \mathrm{~m}$, foram retiradas amostras de madeira, em forma de discos de aproximadamente dois centímetros de espessura, ao longo de $0 \%, 25 \%, 50 \%$ e $75 \%$ da altura total.

Para a determinação do PCS, os discos foram reduzidos em tamanho utilizando-se estilete, sendo esse material seco em estufa de circulação e renovação do ar para obtenção da matéria seca. Após serem retiradas da estufa, foram postas em dessecador para resfriamento e, aproximadamente, meio grama foi utilizado para a determinação do PCS em bomba calorimétrica (C5000, IKA WORKS).

Os dados de PCS foram submetidos à análise estatística pelo software SAS Learning Edition 8.0 (2002), em que se realizou a análise de variância e o teste F. Não havendo diferenças ao longo do fuste ou na interação posição do fuste com espécie (como será apresentado no item resultados e discussões), foi avaliada a quantidade de discos necessária para uma boa amostragem ao longo do fuste para determinação do PCS por espécie. Para tanto, foi utilizado o procedimento de regressão múltipla entre valores médios das amostras individuais nas seguintes combinações de amostragem de disco: somente um $(0 \%, 25 \%, 50 \%$ e $75 \%)$, dois $(0+25 \%, 0+50 \%, 0+75 \%$, $25+50 \%, 25+75 \%$ e $50+75 \%)$ e três $(0+25+50 \%$, $0+25+75 \%, 25+50+75 \%$ e $0+50+75 \%$ ), e a média das amostras individuais de quatro discos $(0+25+50+75 \%)$, partindo do pressuposto que esta é a mais representativa. Dessa maneira, para análise estatística, foram utilizadas como variáveis independentes as combinações de um, dois e três discos, sendo a variável dependente a combinação de quatro discos. Foram realizadas análises de correlação de Pearson e regressão múltipla entre as variáveis envolvidas. A análise de regressão múltipla foi realizada pelo método passo a passo para frente e para trás (stepwise), sendo que a combinação de discos selecionada para o modelo de regressão foi considerada a maneira de melhor amostrar, nas possibilidades de um, dois ou três discos ao longo do fuste. Essas análises foram realizadas no programa SAS 8.0, no subprograma regression, utilizando o procedimento Proc Stepwise.

Para a verificação dos valores observados neste trabalho com outros da literatura, foi realizada comparação de médias de uma amostra com valor fixo através do teste t a $5 \%$ de probabilidade. Para verificar o ajuste dos modelos obtidos, foi realizado o teste de Qui-quadrado a 5\% de probabilidade, para estabelecer a existência de diferenças estatisticamente significativas para o teste. Ainda, foi realizada análise gráfica dos resíduos em valor absoluto, que foram 
calculados como a diferença entre o PCS determinado e os estimado para cada observação.

\section{RESULTADOS E DISCUSSÃO}

A análise de variância não revelou variação significativa do PCS ao longo do fuste das quatro espécies florestais (Tabela 1). Os valores médios foram de $4.482 \mathrm{kcal} \mathrm{kg}^{-1}$ para a Acacia mearnsii, de $4.511 \mathrm{kcal}$ $\mathrm{kg}^{-1}$ para a Mimosa scabrella, de $4.346 \mathrm{kcal} \mathrm{kg}^{-1}$ para o Eucalyptus grandis e de $4.492 \mathrm{kcal} \mathrm{kg}^{-1}$ para o Ateleia glazioveana.

Para Mimosa scabrella, STURION \& TOMASELLI (1990) encontram valores de PCS 4.414kcal $\mathrm{kg}^{-1}$ semelhantes estatisticamente aos observados neste trabalho $\left(4.346 \mathrm{kcal} \mathrm{kg}^{-1}\right)$. No entanto, este valor foi inferior aos médios encontrados por SILVA et al. (1982)e SILVA\& BIASSIO (2009) de 4.700 $\mathrm{kcal} \mathrm{kg}^{-1} \mathrm{e} 4.782 \mathrm{kcal}$ $\mathrm{kg}^{-1}$, respectivamente (Tabela 1). Já para Eucalyptus grandis, o valor de $4.346 \mathrm{kcal} \mathrm{kg}^{-1}$ foi inferior aos verificados por JARA (1989) de $4790 \mathrm{kcal} \mathrm{kg}^{-1}$, por REZENDE (1984), equivalente a $4.805 \mathrm{kcal} \mathrm{kg}^{-1}$ eporVALE et al. (2000), na ordem de $4.641 \mathrm{kcal} \mathrm{kg}^{-1}$. No entanto, o valor de PCS observado para Ateleia glazioveana de $4.492 \mathrm{kcal} \mathrm{kg}^{-1}$ foi semelhante estatisticamente ao encontrado por MATTOS et al. (2000) de $4.637 \mathrm{kcal} \mathrm{kg}^{-1}$ e para Acacia mearnsii, o valor de $4.482 \mathrm{kcal} \mathrm{kg}^{-1}$ foi superior estatisticamente ao valor médio citado por KANNEGIESSER(1990) de 3.744kcal kg-1.
Verificando o potencial energético da madeira das espécies Anadenanthera falcata (Benth.) Speg. (Angico-Branco) e Myracrodruon urundeuva Allemão (Aroeira) em diferentes idades, CINTRA (2009) observou que o poder calorífico superior demonstrou correlação positiva com a idade, ou seja, à medida que aumentou a idade das árvores, também aumentou a energia por unidade de massa. A maioria dos valores inferiores observados para Mimosa scabrella e Eucalyptus grandis, neste trabalho, em relação aos valores da literatura, são em decorrência de que as plantas avaliadas no presente estudo ainda são muito jovens e espera-se que, com o passar do tempo, esse valor tenda a aumentar.

Apesar disso, os valores já se encontram na faixa da média observada em 105 espécies florestais de cinco países tropicais por DOAT (1977), situados entre $4.310 \mathrm{e} 5.170 \mathrm{kcal} \mathrm{kg}^{-1} \mathrm{e}$ também relacionados aos citados por QUIRINO et al. (2005), entre 3.350 a $5.260 \mathrm{kcal}$ $\mathrm{kg}^{-1}$ para a madeira de mais de cem espécies brasileiras. Já os valores semelhantes encontrados para Ateleia glazioveana e superior na Acacia mearnsii indicam que nessas espécies é possível avaliar o potencial energético mesmo quando jovem.

Embora não havendo diferença significativa ao longo do fuste, notam-se variações entre a média e entre o intervalo de confiança para cada posição, principalmente quando se tem na combinação somente de um disco (Figura 1A - 1D). Isso ocorre devido a que algumas propriedades da madeira podem influenciar

Tabela 1 - Resumo da análise de variância e comparação com valores da literatura do poder calorífico de quatro espécies florestais: Acacia mearnsii, Eucalyptus grandis, Ateleia glazioveana e Mimosa scabrella, no município de Frederico Westphalen-RS.

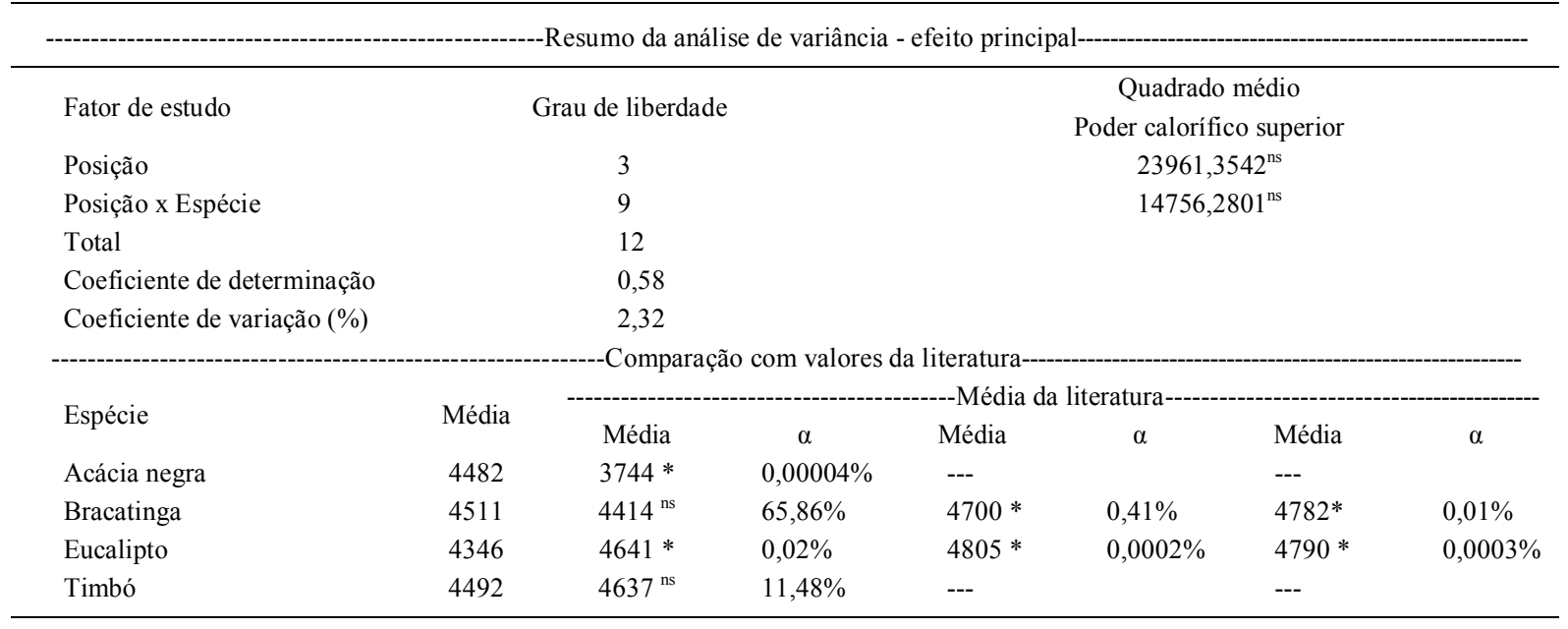

ns = não significativo.

*=significativo a $P>0,05$.

1 = KANNEGIESSER (1990). 2 = STURION \& TOMASELLI (1990). 3 = SILVA et al. (1982). 4= SILVA \& BIASSIO (2009). $5=$ VALE et al. (2000). 6= REZENDE (1984). 7= JARA (1979). 8= MATTOS et al. (2000). 


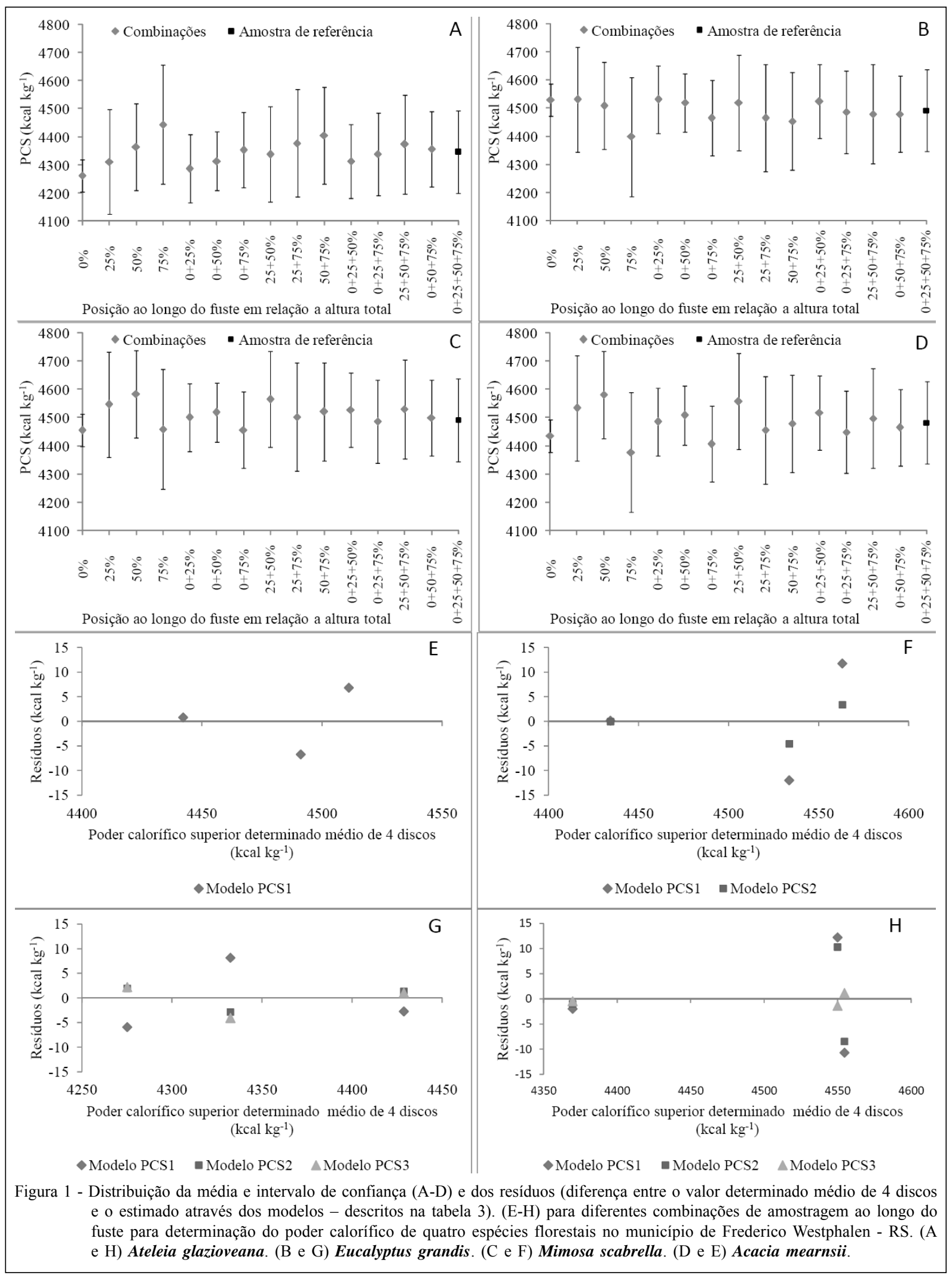

Ciência Rural, v.42, n.9, set, 2012. 
no valor do poder calorífico. Entre elas, a massa específica (GATTO et al., 2003), o teor de lignina, materiais extrativos inflamáveis (óleos, resinas, ceras, etc.) (BURGER \& RICHTER, 1991) e a composição química elementar da madeira (carbono, hidrogênio, nitrogênio, oxigênio e enxofre) (MUNALULA \& MEINCKEN, 2009). A composição química também tem sua importância no teor de cinzas da madeira, pois, à medida que este aumenta, menor é a massa de material orgânico disponível para a combustão, diminuindo, assim, o poder calorífico da madeira. Embora não havendo variação significativa do PCS, numa próxima pesquisa, deve-se também dar preferência a avaliação das variáveis anteriormente citadas ao longo do fuste (massa específica, teor de lignina, materiais extrativos inflamáveis e composição química elementar), com o intuito de verificar correlações na tentativa de explicar as variações que ocorrem.

Conforme observado por SAIDELLES et al. (2010), os teores médios dos macronutrientes na Acacia mearnsii não apresentaram diferenças significativas nas diferentes alturas de amostragem ao longo de fuste, com exceção do $\mathrm{N}$ e do K na madeira, sendo que, quanto mais alta a posição no fuste, maior é o teor do $\mathrm{N}$. Isso é associado à mobilidade desse elemento e, como já destacado, este é um elemento que pode vir a influenciar no valor de PCS. Essas condições vêm possivelmente influenciar no PCS da madeira, tendo em vista que seu valor também é uma função da composição química elementar. Isso sugere que talvez, com o aumento da idade das árvores, haja variação do PCS ao longo do fuste - o que não foi o observado neste trabalho, provavelmente devido ao pequeno tamanho das árvores.

De acordo com os resultados obtidos para estimativa do PCS em relação ao número de discos e à posição ao longo do fuste, para Acacia mearnsii, no mínimo três discos devem ser amostrados para uma boa estimativa, ao passo que, para Mimosa scabrella, dois discos. Já para o Eucalyptus grandis e Ateleia glazioveana, há a possibilidade de amostrar somente um disco (Tabela 2). Como já destacado, embora não ocorrendo variação significativa ao longo do fuste, ao aumentar a composição da amostra com diferentes posições, tende-se a chegar ao valor mais próximo da melhor média para a estimativa do parâmetro PCS, o que pode ser observado na figura 1 (A-D), pois, assim, tende-se a anular os efeitos dos extremos das observações e as influências das propriedades da madeira ligada ao PCS. Além disso, são estas as posições que melhor contribuem para a explicação da média pretendida a ser alcançada, tendo em vista, conforme citam DRAPER \& SMITH (1980), que a regressão múltipla "passo a passo" (Stepwise Multiple Regression) é um dos métodos mais recomendados para uma seleção criteriosa das variáveis explicativas, no estabelecimento de um modelo.

Para Eucalyptus grandis e Ateleia glazioveana, é possível estimar o PCS a partir de um disco, respectivamente, nas posições $50 \%$ e $0 \%$ e, para Mimosa scabrella, com dois discos, nas posições $0+75 \%$, haja vista os altos coeficientes de correlação (Tabela 3) e determinação (Tabela 2) obtidos e o ajuste observado através do teste Qui-quadrado (Tabela 2). Com isso, podem-se diminuir os custos da amostragem, bem como da análise de PCS, conseguindo-se boas medidas, já que o pequeno resíduo obtido, máximo de $\pm 12 \mathrm{kcal} \mathrm{kg}^{-1}$ (Figura 1, E-H), é um valor muito baixo, quando comparado ao valor de PCS médio das espécies de $4.458 \mathrm{kcal} \mathrm{kg}^{-1}$, correspondendo a cerca de $\pm 0,3 \%$.

A diferença das espécies em relação às posições dos discos pode ser explicada num próximo estudo, através da análise da composição elementar ao longo do fuste, visto que a composição química pode vir a influenciar no PCS. Caso haja variação desses elementos em anos posteriores, isso sugere que provavelmente o ponto de amostragem não será o mesmo.

Para serem obtidos valores mais acurados, no mínimo, três discos devem ser amostrados para montagem de uma amostra composta, sendo para Acacia mearnsii, Mimosa scabrella e Eucalyptus grandis nas posições $0 \%, 25 \%$ e $75 \%$ e para Ateleia glazioveana, nas posições $25 \%, 50 \%$ e $75 \%$ em relação à altura total. Nessas posições é que se obtém o valor mais próximo da média composta de quatro discos (partindo do pressuposto de que esta é a mais representativa, como é destacado no item material e métodos), o que pode ser observado na figura 1 e na seleção dos modelos pelo procedimento stepwise (Tabela 2), pois estas são as combinações com maior correlação linear simples com a média de 4 discos, considerada a mais representativa, visto não haver variação significativa ao longo da árvore.

Entretanto, em função dos custos envolvidos para medir o PCS e visando à praticidade, é possível obter uma boa determinação para Eucalyptus grandis e Ateleia glazioveana a partir de um disco, respectivamente, nas posições $50 \%$ e $0 \%$ e, para Mimosa scabrella, com dois discos, nas posições $0+75 \%$. No entanto, ainda no caso de desejo de 
Tabela 2 - Seleção de combinações de amostragem ao longo do fuste, seleção e teste de modelos para estimativa do poder calorífico de quatro espécies florestais: Acacia mearnsii, Mimosa scabrella, Eucalyptus grandis e Ateleia glazioveana, no município de Frederico Westphalen - RS.

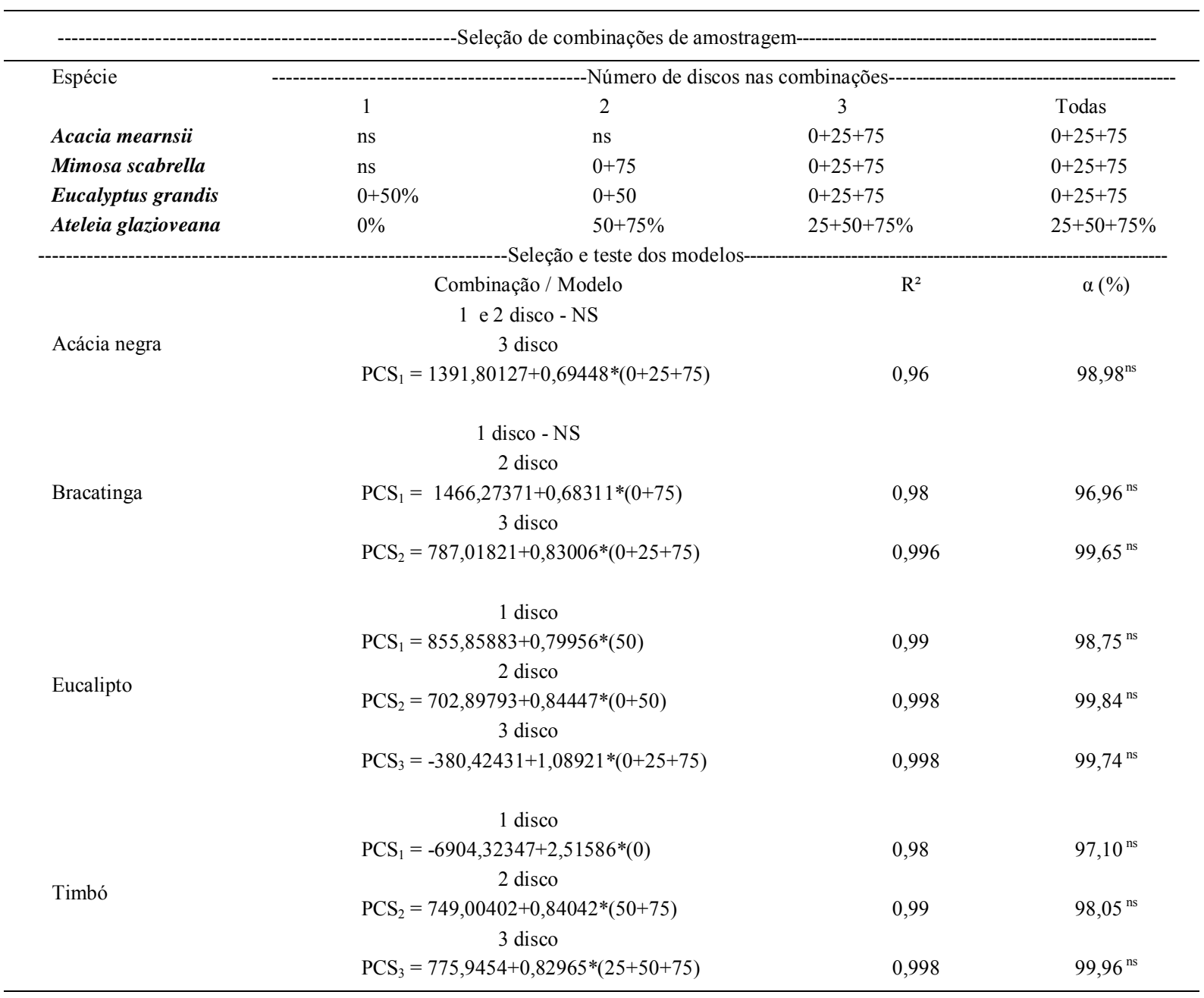

Em que: 1 = valor médio das amostras individuais de discos $(0 \%, 25 \%, 50 \%$ e $75 \%) ; 2=$ valores médios da combinação com dois discos $(0+25 \%, 0+50 \%, 0+75 \%, 25+50 \%, 25+75 \%$ e $50+75 \%) ; 3=$ valores médios da combinação com três discos $(0+25+50 \%, 0+25+75 \%$, $25+50+75 \%$ e $0+50+75 \%)$. Todas = seleção do modelo quando avaliadas todas as combinações possíveis para a amostragem, ou seja, entre 1,2 e $3 . \mathrm{NS}=$ não significativo em nível de tolerância fixado igual a 0,15 . ns = não significativo, o que implica o fato de não existir diferença estatisticamente significativa entre os valores estimados com os modelos e determinados a partir da média de quatro discos para o teste de qui-quadrado.

melhorar tal medida, podem-se utilizar os dados coletados para entrada nos modelos gerados neste trabalho, obtendo-se boas estimativas, visto os baixos resíduos observados e o bom ajuste identificado através do teste Qui-quadrado.

\section{CONCLUSÃO}

Para Eucalyptus grandis e Ateleia glazioveana, é possível estimar o poder calorífico superior a partir de um disco, respectivamente, nas posições 50\% e 0\%, para a Mimosa scabrella com dois discos, nas posições $0+75 \%$ e para Acacia mearnsii, três discos, nas posições $0+25+75 \%$.

Para padronizar o número de amostras ao longo do fuste, recomenda-se, para a Acacia mearnsii, Mimosa scabrella e Eucalyptus grandis, que sejam retiradas no mínimo três discos, respectivamente, nas posições $0 \%$, $25 \%$ e $75 \%$ ao longo do fuste e, para Ateleia glazioveana, nas posições $25 \%, 50 \%$ e $75 \%$ em relação à altura total, para montagem de uma amostra composta. 
Tabela 3 - Coeficientes de correlação de Pearson entre o valor médio de poder calorífico superior de 4 discos e combinações de discos ao longo do fuste para estimativa do poder calorífico de quatro espécies florestais.

\begin{tabular}{|c|c|c|c|c|c|c|c|}
\hline Espécie & Poder Calorífico Superior & \multirow{2}{*}{\multicolumn{6}{|c|}{-------Coeficiente de correlação de Pearson para as combinações------ }} \\
\hline \multirow{7}{*}{ Acacia mearnsii } & \multirow{7}{*}{$\begin{array}{l}\text { Média de } 4 \text { discos } \\
\left(4482 \mathrm{kcal} \mathrm{kg}^{-1}\right)\end{array}$} & & & & & & \\
\hline & & \multicolumn{2}{|l|}{$0 \%$} & $25 \%$ & $50 \%$ & \multicolumn{2}{|r|}{$75 \%$} \\
\hline & & \multicolumn{2}{|l|}{0,740} & $-0,270$ & $-0,170$ & \multicolumn{2}{|r|}{0,550} \\
\hline & & \multirow{2}{*}{$\begin{array}{l}0+25 \\
0,370\end{array}$} & $0+50$ & \multirow{2}{*}{$\begin{array}{c}0+75 \\
0,880\end{array}$} & \multirow{2}{*}{$\begin{array}{r}\text { os------- } \\
25+50 \\
-0,240\end{array}$} & $25+75$ & \multirow{2}{*}{$\begin{array}{r}50+75 \\
0,610\end{array}$} \\
\hline & & & 0,570 & & & 0,680 & \\
\hline & & \multicolumn{2}{|l|}{$0+25+50$} & $0+25+75$ & $25+50+75$ & -------- & $0+50+75$ \\
\hline & & 0,270 & & 0,980 & 0,780 & \multicolumn{2}{|r|}{0,930} \\
\hline \multirow{6}{*}{ Mimosa scabrella } & \multirow{6}{*}{$\begin{array}{l}\text { Média de } 4 \text { discos } \\
\left(4511 \mathrm{kcal} \mathrm{kg}^{-1}\right)\end{array}$} & \multirow{2}{*}{\multicolumn{2}{|c|}{$\begin{array}{c}0 \% \\
0,680\end{array}$}} & $25 \%$ & \multirow{2}{*}{$\begin{array}{c}50 \% \\
0,890\end{array}$} & \multirow{2}{*}{\multicolumn{2}{|c|}{$\begin{array}{c}75 \% \\
0,860\end{array}$}} \\
\hline & & & & 0,930 & & & \\
\hline & & \multirow{2}{*}{$\begin{array}{l}0+25 \\
0,810\end{array}$} & $0+50$ & $0+75$ & \multirow{2}{*}{$\begin{array}{c}25+50 \\
0,920\end{array}$} & $25+75$ & \multirow{2}{*}{$\begin{array}{c}50+75 \\
0,920\end{array}$} \\
\hline & & & 0,760 & 0,990 & & 0,950 & \\
\hline & & \multicolumn{2}{|l|}{$0+25+50$} & $0+25+75$ & $25+50+75$ & & $0+50+75$ \\
\hline & & 0,830 & & 0,998 & 0,970 & & 0,996 \\
\hline & & $0 \%$ & --- & $25 \%$ & $50 \%$ & $\cdots$ & $75 \%$ \\
\hline & & 0,990 & & 0,960 & 0,995 & & $-0,280$ \\
\hline Eucalyptus grandis & Média de 4 discos & $0+25$ & $0+50$ & $0+75$ & os------- & $25+75$ & $50+75$ \\
\hline & & 0,970 & 0,999 & 0,900 & 0,990 & 0,990 & 0,810 \\
\hline & & $0+25+50$ & 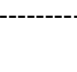 & $0+25+75$ & $25+50+75$ & & $0+50+75$ \\
\hline & & 0,990 & & 0,999 & 0,997 & & 0,970 \\
\hline & & $0 \%$ & & $25 \%$ & $50 \%$ & & $75 \%$ \\
\hline & & 0,990 & & 0,980 & 0,950 & & 0,930 \\
\hline Ateleia glazioveana & Média de 4 discos & $0+25$ & $0+50$ & $0+75$ & $25+50$ & $25+75$ & $50+75$ \\
\hline & & 0,990 & 0,980 & 0,940 & 0,970 & 0,990 & 0,996 \\
\hline & & $0+25+50$ & & $0+25+75$ & 25s-------------- & & $0+50+75$ \\
\hline & & 0,980 & & 0,990 & 0,999 & & 0,995 \\
\hline
\end{tabular}

\section{REFERÊNCIAS}

ABNT (ASSOCIAÇÃO BRASILEIRA DE NORMAS TÉCNICAS). NBR 8633: carvão vegetal - determinação do poder calorífico. Rio de Janeiro, 1994. 13p.

BURGER, L.M.; RICHTER, H.G. Anatomia da madeira. São Paulo: Nobel, 1991. 154p.

CARON, B.O. et al. Resistência inicial de quatro espécies arbóreas em diferentes espaçamentos após ocorrência de geada. Ciência rural, Santa Maria, v.41, n.5, p.817-822, 2011. Disponível em: <http://www.scielo.br/pdf/cr/v41n5/ a948cr4443.pdf>. Acesso em: 02 abr. 2012.
CINTRA, T.C. Avaliações energéticas de espécies florestais nativas plantadas na região do Médio Paranapanema, SP. 2009. 85f. Dissertação (Mestrado em Recursos Florestais) - Escola Superior de Agricultura "Luiz de Queiroz", Piracicaba, SP.

DOAT, J. Le povoir calorifique des bois tropicaux. Revue Bois et Forêts Tropicaux, Nancy, v.172, p.33-48, 1977. Disponível em: <http://bft.cirad.fr/cd/BFT_172_33-55.pdf.>. Acesso em: 18 jul. 2011.

DRAPER, N.R.; SMITH, H. Applied regression analysis. 2.ed. New York: John Wiley \& Sons, 1980. 709p.

GATTO, D.A. et al. Características da lenha produzida na região quarta colônia de imigração italiana do Rio Grande do 
Sul. Ciência Florestal, Santa Maria, v.13, n.2, p.7-16, 2003. Disponível em: <http://www.ufsm.br/cienciaflorestal/artigos/ v13n2/A2V13N2.pdf>. Acesso em: 18 jul. 2011.

JARA, E.R.P. O poder calorífico de algumas madeiras que ocorrem no Brasil. IPT - Comunicação Técnica, São Paulo, v.1797, p.1-6, 1989

KANNEGIESSER, U. Apuntes sobre algunas acacias australianas: 1. Acacia mearnsii De Willd. Ciencia e Investigación Forestal, Concepción, v.4, n.2, p.198-212, 1990.

MALUF, J.R.T. Nova classificação climática do Estado do Rio Grande do Sul. Revista Brasileira de Agrometeorologia, Santa Maria, v.8, n.1, p.141-150, 2000. Disponível em: <http:/ /www.ufsm.br/rba/p14181.html>. Acesso em: 16 jul. 2011.

MATTOS, P.P. et al. Caracterização física, química e anatômica da madeira de Ateleia glazioveana. Colombo: Embrapa Florestas, 2000. 6p. (Comunicado Técnico, 47).

MUNALULA, F.; MEINCKEN, M. An evaluation of South African fuel wood with regards to calorific value and environmental impact. Biomass and Bioernergy, Amsterdam, v.33, n.3, p.415-420, 2009. Disponível em: <http:// www.sciencedirect.com/science/article>. Acesso em: 19 jul. 2011.

PEREIRA, J.C.D.; HIGA, R.C.V. Propriedades da madeira de Taxodium distichum (L.) Rich. Comunicado Técnico, Colombo, n.108, 5p, 2003.

QUIRINO, W.F. et al. Poder calorífico da madeira e de materiais lignocelulosicos. Revista da Madeira, São Paulo, v.89, p.100$106,2005$.

REZENDE, M.A. Uso da técnica de atenuação da radiação gama no estudo da biomassa de Eucalyptus grandis. 1984 74f. Dissertação (Mestrado em Agronomia/Energia Nuclear na Agricultura) - Escola Superior de Agronomia "Luiz de Queiroz", Piracicaba, SP.

SAIDELLES, F.L.F. et al. Determinação do ponto de amostragem para a quantificação de macronutrientes em Acacia mearnsii de Wild. Floresta, Curitiba, v.40, n.1, p.49-62, 2010. Disponível em: <http://ojs.c3sl.ufpr.br/ojs2/index.php/florest a/article/view Article/17098>. Acesso em: 17 jul. 2011.

SAS LEARNING EDITION. Getting started with the SAS Learning Edition. Cary, 2002. 200p.

SILVA, D.A.; BIASSIO, B. Múltiplos usos da Mimosa scabrella Revista da Madeira, São Paulo, n.120, p.23-25, 2009.

SILVA, L.B.X. et al. Estudo comparativo da produção de biomassa para energia entre 23 espécies florestais. In: CONGRESSO FLORESTAL BRASILEIRO, 4., 1982, Belo Horizonte, MG. Anais... São Paulo: Sociedade Brasileira de Silvicultura, 1983. p.872-878.

STURIOM, J.A.; TOMASELLI, I. Influência do tempo de estocagem de lenha de bracatinga na produção de energia. Boletim de Pesquisa Florestal, Colombo, v.21, p.37-47, 1990.

VALE, A.T et al. Produção de energia do fuste de Eucalyptus grandis Hill ex Maiden e Acacia Mangium Willd em diferentes níveis de adubação. Cerne, Lavras, v.6, n.1, p.83-88, 2000. Disponível em: <http://www.dcf.ufla.br/CERNE/artigos/13-0220>. Acesso em: 18 jul. 2011

VALE, A.T. et al. Qualificação e caracterização energética da madeira e casca de espécies do cerrado. Ciência Florestal, Santa Maria, v.12, n.1, p.71-80, 2002. Disponível em: <http:// cascavel.ufsm.br/revistas/ojs-2.2.229>. Acesso em: 18 jul. 2011. 